\title{
¿Transmedia o cross-media? Un análisis multidisciplinar de su uso terminológico en la literatura académica*
}

Received: 29/10/2019 | Revisado: 17/03/2020 | Aceptado: 01/04/2020

DOI: 10.17230/co-herencia.17.33.9

\author{
María Isabel Villa-Montoya** \\ mvilla@eafit.edu.co \\ Diego Montoya-Bermúdez*** \\ diegofer.montoya@javerianacali.edu.co
}

\begin{abstract}
Resumen La distinción entre transmedia y cross-media con frecuencia resulta confusa en los estudios sobre la comunicación. Esta investigación tiene como objetivo revisar el uso de ambos conceptos en la literatura científica publicada en Web of Science y SciELO Citation Index. La investigación parte de una muestra de 895 artículos a los que se les aplica un análisis bibliométrico y un análisis de redes para descubrir las relaciones entre textos. Los resultados del estudio son útiles para conocer la configuración del campo de conocimiento desde una perspectiva que integra las diversas disciplinas implicadas y abren el espectro para entender la comunicación transmedia y cross-media como objetos de estudio afines que deben ser estudiados de forma interdisciplinar.
\end{abstract}

\section{Palabras clave:}

Convergencia, sistemas de comunicación, medios, producción científica, actividad investigativa, transmedia, cross-media, bibliometría, bases de datos académicas.

\section{Transmedia or Crossmedia? A \\ Multidisciplinary Analysis of its Terminological use in the Academic Literature}

\footnotetext{
Abstract The difference between transmedia and crossmedia is frequently found to be confusing in the studies regarding communication. The purpose of this research work is to review the use of both concepts in the scientific literature published in Web of Science and SciELo Citation Index. The research starting point is a sample of 895 articles to which a bibliometric analysis and a network analysis are applied to discover the existing relationships among the texts. The results of the study are useful for knowing the configuration of the knowledge field from a perspective that integrates the diverse disciplines involved,
}

* Este artículo hace parte de los resultados parciales de los proyectos "Sistematización del proyecto MediaLab EAFIT como centro de experimentación en el contexto de la comunicación social" y "Herramienta pedagógica para la creación de series web: Técnicas para la producción audiovisual en contextos transmedia".

** Doctora en Comunicación en la Era Digital. Profesora Titular de la Universidad EAFIT, Medellín, Colombia. ORCID: 0000-00024903-4223

**** Profesor del

Departamento Comunicación y Lenguaje de la Pontificia Universidad Javeriana, Cali, Colombia. ORCID: 0000-0003-2729-6756 
and they open the spectrum to understand the transmedia and crossmedia communication as related objects of study that must be studied in an interdisciplinary manner.

\section{Keywords:}

Convergence, communication systems, media, scientific production, research activity, transmedia, crossmedia, bibliometry, academic database.

El Diccionario de la Real Academia de la Lengua Española no reconoce los términos cross-media o transmedia, aunque con frecuencia han sido utilizados en la literatura académica en español. El Cambridge Dictionary define de forma escueta el concepto de crossmedia en los siguientes términos: "Comunicaciones que involucran más de una forma de comunicación pública". ${ }^{1}$ Llama la atención que el concepto de transmedia no aparezca en este diccionario, como tampoco en el Oxford Dictionary, a pesar de que describe algunas de las características más importantes de la transformación de la comunicación en un escenario convergente.

Aunque desde finales de la década de los setenta del siglo $\mathrm{xx}$ se anuncia la fusión de los medios (Negroponte, 1979), la idea no logra materializarse sino hasta comienzos del siglo XXI cuando, contrario a lo que se pensaba, la digitalización hace que los medios converjan no en una caja negra sino en un sinnúmero de plataformas en las que circulan de un modo desagregado (Jenkins, 2001). La convergencia tiene lugar gracias a un proceso abierto y continuo de intersecciones entre tecnologías de comunicación, industrias y audiencias (Jenkins, 2001). Debido a la proliferación de medios y su naturaleza distribuida, la producción, el contenido y los usos de los medios son fruto de hibridaciones y sinergias con otros medios. En el desplazamiento hacia un análisis particular de estas relaciones intermediales, los conceptos "transmedia" y "cross-media" adquieren un lugar destacado en las investigaciones sobre la comunicación porque aportan claves para comprender procesos amplios en los que se vinculan no solo los medios digitales, sino también los analógicos.

1 En el original: "Communications involving more than one form of public communi-cation" (Cambridge Dictionary, 2011, p. 195). 
Una búsqueda rápida del concepto cross-media en The International Encyclopedia of Communication arroja más de 3000 resultados en los que el término aparece como calificativo que acompaña a otros sustantivos, como los de 'recepción' (Simons, 2014), 'adaptación' (Weedon et al., 2014), 'diseño' (Dena, 2004), 'periodismo' (Singer, 2004) o 'producción' (Bechmann, 2007; Erdal, 2008). A la vez, el concepto de transmedia aparece con frecuencia como calificativo del sustantivo 'narración' (Giovagnoli, 2011; Scolari, 2009). La narración transmedia se ocupa del relato que se distribuye sistemáticamente a través de múltiples canales con el fin de crear una experiencia coordinada y unificada para el usuario. Aquí, cada medio contribuye de forma particular a la historia, es decir, no la reproduce sino que le añade valor. Esta definición, ampliamente extendida por Jenkins (2003a), ha sido revisada, controvertida y actualizada por el propio Jenkins y otros autores que en los últimos años profundizan en diversos aspectos de la convergencia como un proceso abierto y heterogéneo de acuerdo con los contextos donde esta se produce (Gosciola, 2012; Scolari, 2013).

La expansión de los estudios en esta área en las últimas dos décadas ha generado una multitud de trabajos en los que con frecuencia no quedan suficientemente claras las fronteras entre los calificativos cross-media y transmedia. Investigadores como Scolari, Jiménez y Guerrero (2012) se refieren a la narrativa transmedia como un objeto de estudio difícil de situar académicamente por su carácter multidisciplinar, poco delimitado y en permanente transformación. En su esfuerzo por distinguir las fronteras entre ambos, algunas veces se ha entendido la comunicación cross-media como un tipo de estrategia de diversificación propia de las empresas de telecomunicaciones para aprovechar las ventajas competitivas de la distribución multiplataforma (Aarseth, 2006; Bechmann, 2007; Erdal, 2008; Kjus, 2008; Pavlik, 2005; Villa, 2011) porque está claro que la convergencia de medios facilita la promoción a través de fórmulas de adaptación y reutilización del contenido para diferentes medios (Dailey et al., 2005).

Para distinguirse de la comunicación cross-media, Jenkins (2006) declara como condición indispensable de la comunicación transmedia la presentación de elementos únicos en cada plataforma 
que contribuyan a la construcción de un producto final unificado. Esta distinción inaugura de algún modo un debate no resuelto. De acuerdo con Evans (2011), el término transmedia fue usado en primer lugar para referirse a prácticas de promoción que incluían el merchandising o las adaptaciones. En la misma línea, Simons (2014) demuestra con sus investigaciones que los usuarios valoran y frecuentan mucho más las extensiones diegéticas del relato asociado a los dramas televisivos que las expansiones narrativas, y que no son conscientes de muchas de las expansiones narrativas, por lo cual no perciben la narración transmedia de forma unificada. Al contrario de lo que indica Jenkins (2003a), Simons (2014) aclara que para los usuarios no todas las plataformas tienen el mismo valor y que la televisión sigue ocupando un lugar privilegiado en sus consumos transmedia y cross-media.

Este tipo de discrepancias parecen incluso quedarse cortas a la luz de los estudios sobre lo que es un medio y su esencia intermedial, puesto que en su base todos los medios son el resultado de la transferencia de propiedades de muy diversas maneras. Esta apertura del análisis de las relaciones entre textos ha sido abordada previamente al desarrollo de las teorías sobre convergencia con otros conceptos como los de transtextualidad (Genette, 1989), remediación (Bolter y Grusin, 2002), intermedialidad (Elleström, 2017), intertextualidad (Jensen, 1999) o mediatización (Hjarvard, 2009; Krotz, 2008; Livingstone, 2009).

Por eso, antes de dar por cerrada la discusión sobre las diferencias entre transmedia y cross-media con respuestas concluyentes de autores que han querido restringir el uso de los dos términos, aludiendo al uno o al otro como depositario de propiedades exclusivas (Ibrus y Scolari, 2012), es importante revisar los usos académicos de los dos términos y preguntarse hasta qué punto describen fenómenos particulares o compartidos.

Desde esta perspectiva, la investigación parte de la pregunta sobre el uso de los conceptos cross-media y transmedia en la literatura académica con el fin de comprender la naturaleza de los fenómenos de forma amplia y superar los contextos disciplinares en los que han sido empleados.

La investigación utiliza como principal herramienta el análisis bibliométrico mediante el cual es posible analizar cuantitativamente 
un amplio grupo de documentos académicos. Este tipo de análisis permite conocer el uso y el desempeño de los conceptos cross-media y transmedia en la agenda de investigación teniendo en cuenta un extenso rango temporal y espacios geográficos diversos.

En el análisis examinamos indicadores bibliométricos de producción y citación. Estos indicadores arrojan una visión global del crecimiento de la publicación sobre los temas (producción por año), la distribución geográfica del conocimiento (producción por país), la interdisciplinariedad (producción por área) y los principales formatos de publicación (actas de congreso, revistas académicas y libros), además de los temas que están siendo objeto de estudio alrededor de ambos conceptos.

\section{Metodología}

La bibliometría, como rama de la cienciometría, responde a la necesidad de analizar grandes volúmenes de información proveniente de las publicaciones científicas con el fin de identificar los aspectos relativos a la producción, promoción, difusión y aplicación de los conocimientos científico-técnicos (Solano-López et al., 2009). Atendiendo a los procedimientos habituales en bibliometría, esta investigación contabiliza el volumen de la producción e impacto de las publicaciones mediante indicadores de actividad y citación e investigando los vínculos que se han producido entre instituciones de diferentes países, autores y temas. El análisis de citación brinda la posibilidad de identificar afinidad entre grupos de científicos y sus publicaciones, esencial para comprender la estructura profunda de la investigación en un campo disciplinario, escuelas o paradigmas (Garfield, 1985).

Para la selección de la muestra elegimos las bases de datos Web of Science y SciELO Citation Index. La primera, creada por Eugene Garfield, uno de los padres de la bibliometría junto con Derek de Solla Price, presenta condiciones óptimas para este tipo de estudios porque cuenta con más de medio siglo de experiencia y normaliza los datos de sus publicaciones, lo que facilita la exportación y depuración de los resultados (Thomson Reuters, 2008). Web of Science (WoS) contiene información sobre los autores de la publicación, el resumen, 
el título, el número de citaciones, el nombre de la revista en que se publicó, el ISSN, los identificadores de los autores y los conteos de uso, entre otros datos fundamentales para el análisis.

Con el fin de aumentar la cobertura de publicaciones hispanoamericanas, también tuvimos en cuenta la base de datos SciELO Citation Index (SciELO-CI). Esta base de datos busca compensar la baja presencia de revistas latinoamericanas en WoS mediante la visibilización de publicaciones que, aunque no se encuentran a menudo en los primeros lugares del ranquin por su impacto académico, permiten reconocer el trabajo investigativo en América Latina, España, Portugal, el Caribe y Sudáfrica (Repiso et al., 2017).

Los parámetros para la selección de artículos en WoS fueron: (1) búsqueda en la sección principal de la base de datos; (2) índices SCI-EXPANDED, SSCI, A\&HCI, CPCI-S, CPCI-SSH, BKCI-S, BKCI-SSH, ESCI, CCR-EXPANDED, IC; (3) período de tiempo: sin filtro, teniendo como fecha de corte por búsqueda el 18 de julio de 2017; (4) búsqueda por tema (título + palabras clave + abstract), y (5) términos: transmedia y cross-media. Como resultado encontramos 354 publicaciones asociadas a transmedia y 514 publicaciones recuperadas con el término cross-media en WoS.

Por su parte, en SciELO-CI, la ecuación de búsqueda contempló: (1) término de búsqueda (tema): transmedia, cross-media, y (2) período de tiempo: sin filtro, teniendo como fecha de corte por búsqueda el 18 de julio de 2017. Este procedimiento arrojó 24 resultados asociados a transmedia y 3 a cross-media. En total, la muestra del estudio la conforman 895 artículos que cumplen con las características antes señaladas en ambas bases de datos. Todos los datos estadísticos relacionados con las características de la producción científica fueron analizados en Excel.

Para completar el análisis, hicimos una revisión posterior de temas por medio de análisis y la visualización de redes con ayuda del programa Pajek. Las redes proporcionan una imagen visual de las relaciones en un campo de conocimiento y la proximidad, a veces imperceptibles entre un texto y otro (Lievrouw, 1989). El análisis de redes suministra información gráfica de los vínculos en las investigaciones teniendo en cuenta las múltiples relaciones que pueden surgir de la complejidad (Molina, 2009). Con esta metodología 
se describe la forma en la que están vinculados los temas estudiados y se hace posible identificar cómo se relaciona cada publicación con otra de la estructura (Sanz Méndez, 2003). Con el análisis de redes situamos las instituciones educativas que comparten intereses por su proximidad con el tema, su centralidad y los asuntos sobresalientes en los estudios vinculados a los términos cross-media y transmedia.

Las redes de temas se construyeron a partir de las palabras clave en el conjunto de la muestra. Los nodos por palabra clave suministran una representación visual de las conexiones entre los artículos. Para realizar este ejercicio, en primer lugar, tomamos la base de datos de las palabras clave normalizadas del total de la muestra y eliminamos del listado las palabras que aparecían una sola vez, quedando aquellas que redundan en varias investigaciones. En segundo lugar, construimos un archivo relacional en texto plano con los títulos de las publicaciones con un código único asignado. Este archivo fue procesado con el software Pajek, el cual facilita la transformación de los artículos en relaciones visuales de acuerdo con el comando ejecutado.

En las redes eliminamos las relaciones débiles, entendidas como palabras que coincidían solo una vez en los artículos y otorgamos a cada nodo una dimensión gráfica según la cantidad de relaciones que presentaba. De este modo, los nodos de las palabras más conectadas adquieren un mayor tamaño. Finalmente, por medio del algoritmo Louvain generamos las agrupaciones de las palabras afines.

Este ejercicio se realizó en dos redes de temas, una para los artículos sobre transmedia y otra para los artículos sobre crossmedia. En las gráficas, los puntos o nodos representan las palabras clave de las publicaciones, las líneas expresan las conexiones entre publicaciones, el tamaño de los nodos hace posible visualizar la cantidad de conexiones que poseen, y los colores muestran la agrupación por clústeres de palabras con un vínculo más estrecho. 


\section{Resultados}

\section{Volumen de publicaciones que acogen los términos cross-media y transmedia}

La producción académica en torno a los estudios que incluyen los términos transmedia y cross-media ha mantenido un interés creciente en las últimas dos décadas. Sin embargo, la producción académica que emplea el término transmedia es inferior a la que utiliza el término cross-media. Encontramos 354 publicaciones asociadas a transmedia y 512 a cross-media. Es importante notar que esta diferencia no se aprecia en SciELO-CI, donde, al contrario de lo que ocurre en WoS, vemos solo tres publicaciones que utilizan el término cross-media y 24 que emplean el término transmedia, lo que indica una configuración del campo de conocimiento alrededor de los problemas que atañen a la cross-medialidad muy débil en el Cono Sur.

El concepto de transmedia comienza a ser objeto de publicación en el 2004, mientras que las publicaciones sobre cross-media son visibles desde 1993. Parece evidente que los fenómenos propios de la digitalización y la convergencia han incentivado una gran parte de los estudios sobre comunicación transmedia, mientras que la investigación sobre procesos cross-media puede responder a escenarios previos a la digitalización de los medios.

Cuando profundizamos en el tipo de documentos recuperados vemos que $71 \%$ de las referencias asociadas al concepto de transmedia son artículos académicos y solo $11 \%$ proviene de actas de congresos; mientras que la mayoría de las publicaciones sobre cross-media encontradas son actas de congresos (52\%) y $44 \%$ son artículos. Estas diferencias nos manifiestan una configuración del campo científico y la difusión del conocimiento a través de formatos académicos diversos.

\section{Origen de la producción científica}

La mayor parte de la producción científica encontrada en la búsqueda del concepto de transmedia se concentra en tres países: Estados Unidos de América (26 \%), España (24 \%) e Inglaterra (8\%). Los demás países aportan entre un $4 \%$ y un $2 \%$ al total de publicaciones 
encontradas. El análisis alrededor del término cross-media indica, por el contrario, que la mayor parte de las publicaciones que tratan este tema proviene de la República Popular China (26 \%) seguidas por las de Estados Unidos de América (17 \%), Inglaterra (8 \%), Japón (6 \%), Alemania (6 \%) e Italia (5 \%). Llama la atención que España, un país representativo en la investigación alrededor del concepto transmedia, figure con tan solo 0,03 \% de la producción académica registrada en WoS y SciELO-CI en torno al término cross-media.

Un $98 \%$ de la producción académica alrededor del término cross-media está escrita en inglés, mientras que $23 \%$ de las publicaciones que se encontraron sobre transmedia se presenta en español. Estos datos son coherentes con el papel destacado de España en la producción en torno al término transmedia. En este campo se destacan la Universidad Pompeu Fabra, la Universidad Complutense de Madrid, la Universidad Autónoma de Barcelona y la Universidad Central de Cataluña. Es importante anotar que esta fuerte presencia de España como polo de producción académica sobre los estudios transmedia se debe en gran medida al trabajo que ha realizado el profesor Carlos Scolari, quien es uno de los referentes hispanoamericanos más prolíficos en lo que concierne a la investigación sobre las narrativas transmedia.

A diferencia de lo que ocurre alrededor del concepto de crossmedia, en los diez países con mayor producción -que incluyen el término transmedia en el título, el resumen y las palabras clave- no figura ningún asiático. Por el contrario, se identifican algunos países de América Latina como Colombia (0,02 \%) y Brasil (0,02 \%) con una producción incipiente sobre el tema.

También llama la atención que si bien China es el mayor productor de publicaciones con el término cross-media, si se limita la búsqueda al campo de la comunicación esta no tiene un papel representativo. Algo similar se observa en Japón. En ambos países asiáticos el concepto cross-media es distintivo en las ingenierías y las ciencias computacionales, mientras que el uso del término crossmedia sobresale en los estudios de comunicación en Estados Unidos, Holanda, España y Dinamarca. 


\section{Red geográfica de construcción de conocimiento}

En las siguientes imágenes es posible identificar los países con mayor número de publicaciones sobre el tema, teniendo en cuenta la procedencia de los autores de las referencias analizadas y sus vínculos como coautores de un mismo artículo.

\section{Imagen 1}

Red geográfica de construcción de conocimiento en torno al concepto transmedia

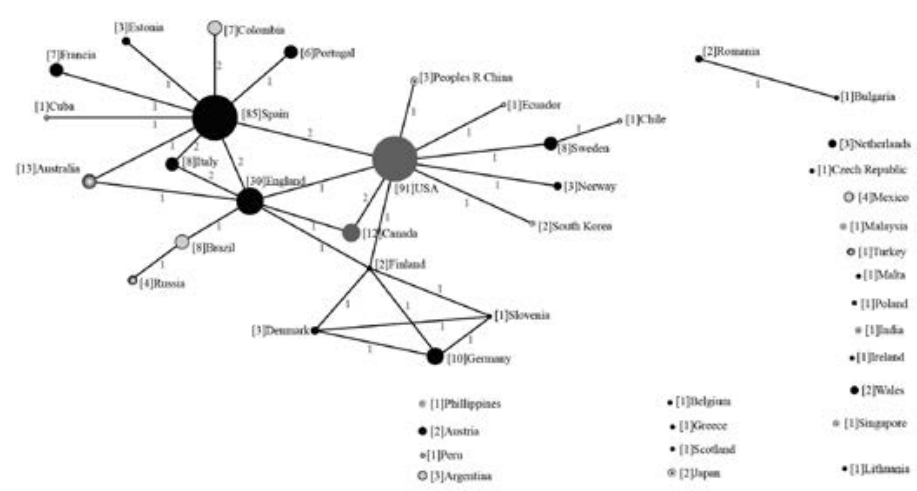

Fuente: Elaboración propia.

En relación con el concepto transmedia, la red muestra cómo este presenta dos grandes nodos de producción académica concentrados en Estados Unidos y España, con una evidente influencia de este último país en la producción que se realiza en otros contextos como el latinoamericano y el europeo, con especial énfasis en los países con lenguas romances. Por su parte el nodo estadounidense, que también tiene influencia en Europa en los países que tienen el inglés como lengua principal, genera un impacto importante en la producción de algunos países asiáticos.

Se destaca en esta red lo que podríamos catalogar como dos niveles adicionales en la producción: el inglés -con 30 productos- y el australiano, canadiense y alemán, con una producción entre 10 y 13 artículos. Cabe también resaltar en la red de producción transmedia cómo Brasil y Colombia se convierten en nodos dentro de la región de Suramérica con un promedio de 7 artículos por país. 


\section{Imagen 2}

Red geográfica de construcción de conocimiento en torno al concepto cross-media

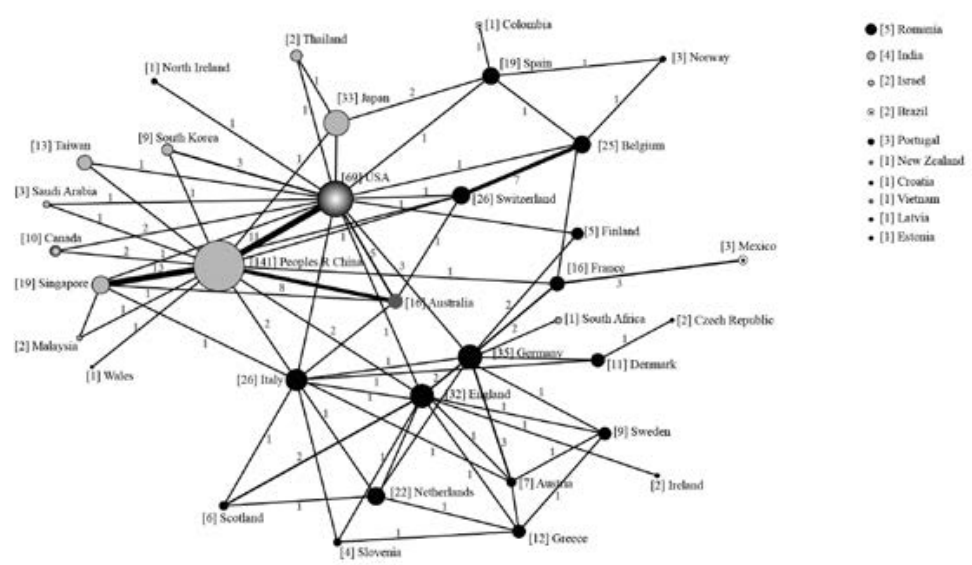

Fuente: Elaboración propia.

La red revela tres nodos de producción académica, el primero alrededor de la República Popular China, el segundo en torno a Estados Unidos de América, el tercero ubicado en Alemania, seguido por Japón e Inglaterra y otros países europeos con un peso relativamente similar, como Bélgica, Noruega o Italia. La imagen expone en cada país el número de artículos encontrados, y el espesor de la línea expresa qué tan fuerte es la relación entre países debido a la colaboración de autores de diversas procedencias. Como se puede observar, es escasa la participación de los países latinoamericanos en la red. Solo aparecen Colombia y México, el primero con un artículo en coautoría con España y el segundo con un artículo en coautoría con Francia.

\section{Áreas de publicación}

Las áreas registradas corresponden a las revistas en que fueron publicados los registros analizados. Esta asignación es producto de la revisión de WoS de acuerdo con el campo al que cada revista se encuentra suscrita, pero en ningún caso responde a los temas de los artículos específicos que podrían ser objeto de una discusión más amplia, que excede el alcance de esta investigación. Las tablas 1 y 2 muestran los campos donde los conceptos transmedia y cross-media han sido más utilizados. 


\section{Tabla 1}

Áreas de conocimiento donde se suscribe la investigación asociada a los conceptos transmedia y cross-media

\begin{tabular}{|c|c|c|c|}
\hline $\begin{array}{l}\text { Área de conocimiento } \\
\text { Transmedia }\end{array}$ & $\%$ & $\begin{array}{c}\text { Área de conocimiento } \\
\text { Cross-media }\end{array}$ & $\%$ \\
\hline Communication & $44,35 \%$ & Computer Science & $54,30 \%$ \\
\hline Film Radio Television & $18,64 \%$ & Engineering & $29,05 \%$ \\
\hline Computer Science & $11,02 \%$ & Communication & $11,14 \%$ \\
\hline Educational Research & $9,04 \%$ & $\begin{array}{l}\text { Imaging Science } \\
\text { Photographic Technology }\end{array}$ & $10,86 \%$ \\
\hline Cultural Studies & $8,47 \%$ & Telecommunications & $9,45 \%$ \\
\hline Literature & $6,72 \%$ & Business Economics & $7,05 \%$ \\
\hline $\begin{array}{l}\text { Arts Humanities other } \\
\text { Topics }\end{array}$ & $5,93 \%$ & $\begin{array}{l}\text { Environmental } \\
\text { Sciences Ecology }\end{array}$ & $5,22 \%$ \\
\hline Business Economics & $4,23 \%$ & $\begin{array}{l}\text { Information Science / } \\
\text { Library Science }\end{array}$ & $3,67 \%$ \\
\hline $\begin{array}{l}\text { Information Science / } \\
\text { Library Science }\end{array}$ & $3,95 \%$ & Optics & $2,92 \%$ \\
\hline Social Sciences other Topics & $3,39 \%$ & $\begin{array}{l}\text { Education / } \\
\text { Educational Research }\end{array}$ & $2,54 \%$ \\
\hline
\end{tabular}

Fuente: Elaboración propia a partir de datos provenientes de la WoS y SciELO-CI.

Como se observa en la tabla 1, los dos conceptos tienen una representación muy heterogénea en las áreas de conocimiento. Mientras que la comunicación y los estudios de radio y televisión concentran la mayor parte de investigaciones que usan el término transmedia, las ciencias de la computación y las ingenierías optan por el uso del concepto cross-media. Es importante notar que nueve de las diez áreas destacadas en torno al término transmedia son del ámbito cultural y social, mientras que cinco de las diez áreas más destacadas que usan el término cross-media presentan un interés común por los aspectos técnicos. 


\section{Autores y títulos más referenciados}

Observamos dos campos de estudio muy distintos en cuanto a las fuentes de conocimiento. No se identifican concurrencias aparentes en los autores que inspiran o fundan los estudios que tienen como eje los términos transmedia y cross-media. Sin embargo, el análisis particular de los resultados provenientes del área de la comunicación revela un alto grado de coincidencias en los autores que se referencian en las publicaciones de los dos conceptos.

\section{Tabla 2}

Autores más referenciados en torno a los conceptos transmedia y cross-media

\begin{tabular}{l|l|l|l}
\hline \multicolumn{1}{c|}{ Transmedia } & \multicolumn{2}{c}{ Cross-media } \\
\hline Autor & $\begin{array}{l}\text { Número de } \\
\text { referencias }\end{array}$ & \multicolumn{1}{c}{ Autor } & $\begin{array}{l}\text { Número de } \\
\text { referencias }\end{array}$ \\
\hline Henry Jenkins & 473 & Yi Yang & 112 \\
\hline Carlos Alberto Scolari & 175 & Beat Signer & 77 \\
\hline Christy Dena & 50 & Fei Wu & 52 \\
\hline Marie Laure Ryan & 49 & Yueting Zhuang & 52 \\
\hline Marsha Kinder & 45 & Pierfrancesco Bellini & 41 \\
\hline Manuel Castells & 39 & Hong Zhang & 40 \\
\hline Elizabeth Evans & 33 & Jiwoon Jeon & 37 \\
\hline Lev Manovich & 32 & Nikhil Rasiwasia & 37 \\
\hline Jason Mittell & 30 & Meng Wang & 31 \\
\hline Matt Hills & 27 & David G. Lowe & 29 \\
\hline
\end{tabular}

Fuente: Elaboración propia a partir de datos provenientes de la WoS y SciELO-CI. 


\section{Tabla 3}

Autores más referenciados en torno a los conceptos transmedia y crossmedia en el campo de la comunicación

\begin{tabular}{l|l|l|l}
\hline \multicolumn{1}{c|}{ Transmedia en comunicación } & \multicolumn{2}{c}{ Cross-media en comunicación } \\
\hline Autor & $\begin{array}{l}\text { Número de } \\
\text { referencias }\end{array}$ & \multicolumn{1}{c}{ Autor } & $\begin{array}{c}\text { Número de } \\
\text { referencias }\end{array}$ \\
\hline Henry Jenkins & 253 & Henry Jenkins & 30 \\
\hline Carlos Alberto Scolari & 119 & Ivar John Erdal & 14 \\
\hline Manuel Castells & 22 & Nick Couldry & 12 \\
\hline Marsha Kinder & 22 & John Dimmick & 11 \\
\hline Arnau Gifreu Castells & 22 & Sonia Livingstone & 11 \\
\hline Elizabeth Evans & 21 & Hasebrink Uwe & 11 \\
\hline Christy Dena & 20 & Klaus Bruhn Jensen & 10 \\
\hline Jonathan Gray & 17 & Kim Christian Schrøder & 8 \\
\hline Sonia Livingstone & 14 & Carlos Alberto Scolari & 7 \\
\hline Robert E. Howard & 13 & Pablo J. Boczkowski & 7 \\
\hline
\end{tabular}

Fuente: Elaboración propia a partir de datos provenientes de la WoS y SciELO-CI.

En lo referido al campo de la comunicación es pertinente subrayar la manera como se han utilizado ambos conceptos y quiénes son sus principales referentes. La tabla 3 da una clara indicación de cómo en los estudios de la comunicación ambos son tomados como conceptos muy cercanos y en algunas oportunidades hasta como sinonimias, de ahí que Henry Jenkins termine siendo la fuente principal en ambas nociones.

Asimismo, llama especialmente la atención cómo, cuando comparamos las tablas 2 y 3 en lo relacionado con el campo transmedial, siete de los diez autores referenciados se mantienen cuando se ubica el concepto en todas las áreas y cuando se profundiza en el campo de la comunicación. No obstante, al hacer la misma comparación entre las tablas 2 y 3 respecto a la noción cross-media, ningún autor de los diez más referenciados en el concepto cross-media aparece entre los diez autores del mismo concepto en el entorno de la comunicación, lo que da una clara lectura de cómo dicho concepto tiene diversidad de enfoques, como se observa en la tabla 4. 


\section{Tabla 4}

Títulos más citados en torno al concepto transmedia en el campo de la comunicación

\begin{tabular}{l|c}
\hline \multicolumn{1}{c|}{ Autor } & Formato \\
\hline Henry Jenkins (2006), Convergence Culture. & Libro \\
\hline $\begin{array}{l}\text { Henry Jenkins (2003a), "Transmedia Storytelling: Moving characters } \\
\text { from books to films to video games can make them stronger and more } \\
\text { compelling". }\end{array}$ & Artículo \\
\hline Carlos Alberto Scolari (2013), Narrativas transmedia. & Libro \\
\hline $\begin{array}{l}\text { Carlos Alberto Scolari (2009), "Transmedia Storytelling: Implicit } \\
\text { Consumers, Narrative Worlds, and Branding in Contemporary Media } \\
\text { Production". }\end{array}$ & Artículo \\
\hline $\begin{array}{l}\text { Marsha Kinder (1991), Playing with Power in Movies, Television and } \\
\text { Video Games. }\end{array}$ & Libro \\
\hline $\begin{array}{l}\text { Henry Jenkins, Sam Ford, \& Joshua Green (2013), Spreadable media: } \\
\text { creating value and meaning in a networked culture. }\end{array}$ & Libro \\
\hline $\begin{array}{l}\text { Elizabeth Evans (2011), Transmedia television: Audiences, New Media } \\
\text { and Daily Life. }\end{array}$ & Libro \\
\hline \begin{tabular}{l} 
Christy Dena (2008), "Emerging Participatory Culture Practices". \\
\hline $\begin{array}{l}\text { Jonathan Gray (2010), Show Sold Separately: Promos, Spoilers, and Other } \\
\text { Media Paratexts. }\end{array}$
\end{tabular} & Artículo \\
\hline $\begin{array}{l}\text { Geoffrey Long (2007), Transmedia Storytelling: Business, Aesthetics and } \\
\text { Production at the Jim Henson Company. }\end{array}$ & Tesis \\
\hline
\end{tabular}

Fuente: Elaboración propia a partir de datos provenientes de la WoS y SciELO-CI.

Las relaciones encontradas en el área de comunicación en torno a los conceptos de transmedia y cross-media se derivan en gran parte del discurso de la convergencia, en cuanto condición que describe la circulación de contenidos a través de múltiples sistemas mediáticos. El trabajo de Jenkins (2006) ocupa el lugar más destacado en los estudios referenciados en el campo. Recogiendo trabajos previos y su definición de comunicación transmedia (2003a), Jenkins sitúa la transformación digital como un fenómeno que pone en entredicho las estructuras técnicas, culturales y sociales que han rodeado a los medios especialmente norteamericanos. Es sobresaliente que no sea la comunicación transmedia propiamente dicha sino la participación el principal objeto de estudio del texto, a menudo tratada como un tema subsidiario en las investigaciones que refieren el trabajo de Jenkins. 
El segundo lugar que ocupa el artículo "Transmedia Storytelling: Moving characters from books to films to video games can make them stronger and more compelling" (Jenkins, 2003a) en los estudios alrededor del término transmedia es también relevante. Una buena parte de los trabajos recuperados a través de las búsquedas sobre el concepto transmedia en comunicación tienen como principal preocupación la narrativa en un entorno multiplataforma.

\section{Tabla 5}

Títulos más citados en torno al concepto cross-media en el campo de la comunicación

\begin{tabular}{l|l}
\hline \multicolumn{1}{c|}{ Autor } & Formato \\
\hline Henry Jenkins (2006), Convergence Culture. & Libro \\
\hline $\begin{array}{l}\text { Carlos Alberto Scolari (2009), "Transmedia Storytelling: Implicit } \\
\text { Consumers, Narrative Worlds, and Branding in Contemporary Media } \\
\text { Production". }\end{array}$ & Artículo \\
\hline $\begin{array}{l}\text { Ivar John Erdal (2009), "Cross-Media (Re)Production Cultures". } \\
\text { Nick Couldry (2007), Consumption and Public Life. }\end{array}$ & Artículo \\
\hline $\begin{array}{l}\text { Uwe Hasebrink, \& Hannah Domeyer (2012), "Media repertoires as } \\
\text { patterns of behaviour and as meaningful practices". }\end{array}$ & Libro \\
\hline $\begin{array}{l}\text { Henry Jenkins et al. (2013), Spreadable media: creating value and } \\
\text { meaning in a networked culture. }\end{array}$ & Libro \\
\hline $\begin{array}{l}\text { Herbert E. Krugman (1971), "Brain waves measures of media } \\
\text { involvement". }\end{array}$ & Artículo \\
\hline $\begin{array}{l}\text { Prasad A. Naik (2003), "Understanding the Impact of Synergy in } \\
\text { Multimedia Communications". }\end{array}$ & Artículo \\
\hline \begin{tabular}{l} 
Bruns Axel (2008), Blogs, Wikipedia, Second Life, and Beyond. \\
\hline $\begin{array}{l}\text { Ivar John Erdal (2011), "Coming to Terms with Convergence } \\
\text { Journalism: Cross-Media as a Theoretical and Analytical Concept". }\end{array}$
\end{tabular} & Libro \\
\hline
\end{tabular}

Fuente: Elaboración propia a partir de datos provenientes de la WoS y SciELO-CI.

Algo similar a lo que sucede en los estudios sobre lo transmedia ocurre con los recuperados con el concepto cross-media (tabla 5). Al contrario de lo que algunos autores afirman en relación con el uso de la palabra cross-media en los artículos que no tienen como objeto de estudio la narración, en el segundo lugar de las obras más referenciadas se encuentra el artículo de Scolari (2009) "Transmedia 
Storytelling: Implicit Consumers, Narrative Worlds, and Branding in Contemporary Media Production". En este texto aparecen muestras de una relación estrecha entre los términos transmedia y cross-media. Si bien el autor emplea la expresión "concepto adyacente" (p. 587) para referirse a la noción cross-media, lo sitúa en "la misma galaxia semántica” (p. 588), con otros que aluden a una misma experiencia y práctica productiva combinatoria de medios o plataformas.

A partir de este texto se observa la aplicación de la noción cross-media en un terreno fértil y muy amplio, y mientras solo un grupo de autores se mantiene plegado al problema narrativo, otros exploran distintos caminos marcados por autores europeos, como los del noruego Erdal (2009), preocupado por las formas de producción de contenido. A diferencia de los estudios en torno a la comunicación transmedia, centrados en la ficción, Erdal se ocupa del periodismo y de lo que ocurre en las salas de redacción que deben producir noticias para distintas plataformas.

Las discusiones acerca de lo que puede o no ser entendido como medio y su papel en la configuración fluida de la esfera pública y privada (Couldry et al., 2007) son además un pilar importante en los estudios de comunicación que identificamos en el análisis en torno al término cross-media. También venido de Europa, en este caso de Alemania, el trabajo de Hasebrink y Domeyer (2012) inspira un gran número de investigaciones sobre comunicación cross-media utilizando el concepto de repertorio de medios, con el cual se observa el comportamiento de los sujetos ante el uso de distintas tecnologías de forma integrada en espacios particulares.

\section{Análisis de redes}

Construir las redes de temas para ambos conceptos permite verificar cómo las nociones transmedia y cross-media son afectadas por sus campos de origen. La red en torno al tópico transmedia visualiza cómo a partir del concepto "transmedia storytelling" expuesto por Jenkins en 2003 se deriva la mayoría de los estudios sobre el concepto transmedia y cómo todos estos en su totalidad (incluyendo las redes que se alejan del núcleo storytelling) aluden al campo de los estudios en comunicación, ya sea desde aspectos narrativos, mediáticos, de género, o en estudios de caso. 
Imagen 3. Red de temas en torno al concepto transmedia

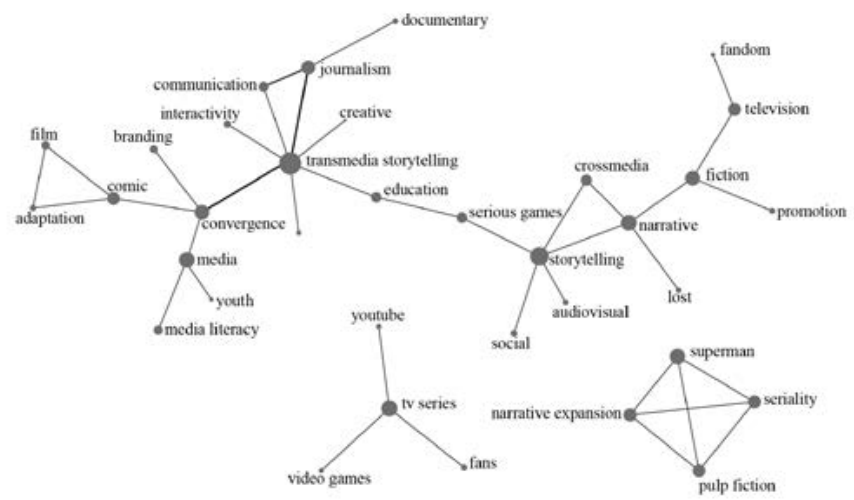

Fuente: Elaboración propia.

Opuesto a lo anterior en la red de temas del concepto crossmedia (imagen 4), en la que se evidencian ocho subredes en torno a campos distintos, lo cual demuestra lo que hemos anunciado desde el principio: que el concepto cross-media -que para los estudiosos de la comunicación nos llegó de la mano de la noción transmedia, y enfocada netamente al estudio de los medios- tiene orígenes anteriores y diversos en campos que lo han explorado desde distintas perspectivas.

Imagen 4. Red de temas en torno al concepto cross-media

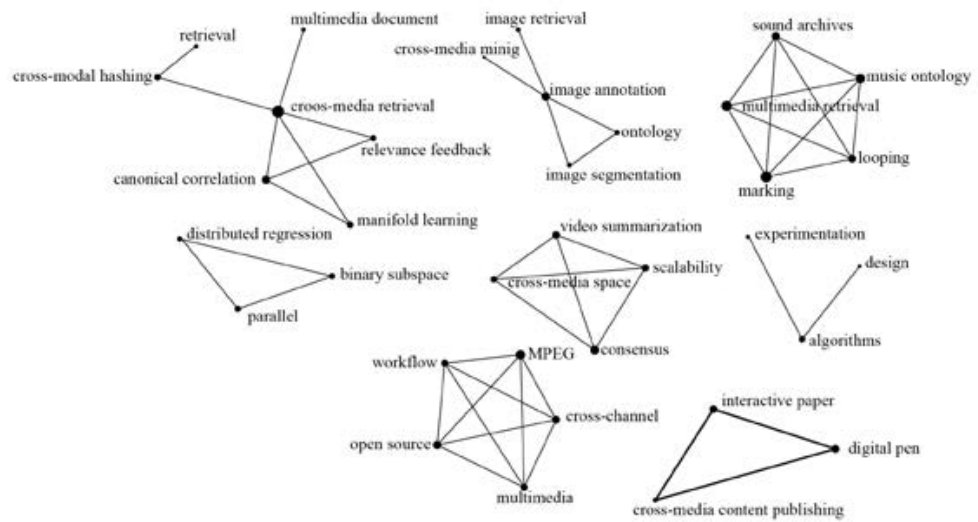

Fuente: Elaboración propia. 


\section{Discusión}

La investigación alrededor de los conceptos transmedia y crossmedia presenta un amplio y nutrido cuerpo de trabajos y perspectivas, como lo demuestran los datos recogidos hasta ahora. De acuerdo con Hasebrink y Hepp (2017), la idea de cross-medialidad resulta esencial en un entorno profundamente mediatizado para comprender la manera en que los medios se ensamblan en el terreno individual y social. Partiendo de la perspectiva de Hasebrink y Hepp (2017) podemos decir que es necesario comprender los medios como parte de una estructura y combinar perspectivas, en nuestro caso disciplinares, para comprender en amplio sentido cómo se configuran los medios. En una primera aproximación identificamos cinco áreas que desde diversas miradas atienden la cross-medialidad: la computación, la comunicación, la administración, la bibliotecología o las ciencias de la información y la educación. Cada una de ellas resulta fundamental para concebir las características de la cross-medialidad como un área que observa problemas relacionados con: (1) la publicación y la recuperación de contenidos mediante múltiples medios: ello implica concebir asuntos como la reproducción en diversas pantallas, la escalabilidad, la agrupación de datos dispersos en varios medios, la interoperabilidad o las correlaciones semánticas; (2) los sistemas de comunicación capaces de ensamblar o conectar distintos medios desde la perspectiva de la producción, el contenido, la recepción o el consumo; (3) las estrategias económicas dirigidas a administrar, ampliar o diversificar productos, medios y públicos por medio de la creación de sistemas de empaquetamiento de datos, reutilización y mercadeo cruzado; y (4) la asociación de contenidos que pueden ser vinculados semánticamente por su relación entre ellos para facilitar el aprendizaje multimodal, colectivo y participativo.

En lo referente a la noción sobre lo transmedial, los resultados obtenidos en la investigación ponen de relieve el valor de la comunicación como el área que más se ha apropiado de la noción, con más de un 70 \% de la producción académica. Es importante aclarar que en este caso se incluyeron áreas cercanas a la comunicación y los estudios de medios como cine, radio y televisión, además de los estudios culturales, como campos de trabajo en los cuales se ha 
empleado dicha noción. Precisamente en el área de la comunicación destacan los enfoques semióticos, narrativos e intermediales, de ahí que se explique por qué la mayor parte de la producción académica en comunicación viene acompañada por el sustantivo 'narración'.

Al profundizar en el área de la comunicación, la narración transmedia además de haber aportado un amplio repertorio de investigaciones en el ámbito de la comunicación de medios y el entretenimiento, ha permeado otras subáreas como el periodismo (Ford, 2007; Peñafiel, 2016; Renó, 2011), el marketing (Cronin, 2016; Hills, 2016), e inclusive el eduentretenimiento (Wohlwend, 2012). Otra área que empieza a perfilarse en la investigación en términos transmediales es la educativa, que alcanza casi un $10 \%$ de la producción académica. Para el caso del fenómeno educativo, el trabajo sobre la transmedialidad se enfoca en el uso de diferentes medios al interior de las aulas de clase. Al igual que en los estudios sobre la comunicación, en el área educativa la transmedialidad es vista como la posibilidad de generación de narraciones y creación de contenidos dentro del aula de clase por parte de los estudiantes, como lo plantean Scolari, Lugo y Masanet (2019).

Los resultados obtenidos en esta exploración rebaten la idea de la cross-medialidad como un procedimiento con menores posibilidades que la transmedialidad, en el que un contenido se replica de forma idéntica en diferentes medios (Veglis, 2012). Encontramos una amplia variedad de referentes que sitúan la cross-medialidad como un problema en el área de la comunicación que supera la emisión multiplataforma para interrogarse por el márquetin, (Okazaki et al., 2012), la adaptación (Weedon et al., 2014), el aprendizaje (Wu et al., 2015) o la recepción en un entorno convergente (Simons, 2014).

A pesar de las diferencias encontradas entre las diversas perspectivas y áreas, observamos como objetivos predominantes en la literatura académica sobre los conceptos cross-media y transmedia la mezcla, la coherencia y la continuidad, y como problemas comunes la migración y la integración. La distinción entre los contenidos, así como la posibilidad de ofrecer diversos puntos de entrada a una misma historia, parecen ser condiciones necesarias para referirse a la transmedialidad y la cross-medialidad como un sistema que supera el acomodo de un contenido para su emisión por diversos medios. 
La manera en que se emplean los términos en la literatura de la muestra parece responder a la configuración particular de los colegios invisibles, entendidos como una red de autores conectados a través de citaciones (De Solla, 1965), y definida en este caso en gran medida por el origen de los autores y su filiación institucional.

Si tenemos en cuenta que la mayor parte de la investigación sobre cross-media se ha realizado en países asiáticos, con una muy baja publicación de autores provenientes de países hispanohablantes y, en cambio, la investigación sobre transmedia sobresale en España y Estados Unidos, es predecible que los conceptos se hayan esparcido de forma diferente, aludiendo en muchos casos al mismo fenómeno con dos perspectivas que en lugar de presentarse como miradas enfrentadas del problema son complementarias, pero poco articuladas.

\section{Conclusiones}

Esta investigación tuvo como objetivo identificar el uso de los conceptos cross-media y transmedia en la literatura académica. Al respecto se encontró que la noción de cross-medialidad cuenta con un volumen mayor de publicaciones académicas y es más antigua. La publicación académica sobre ambos conceptos presenta un origen diverso. Todos los estudios sobre transmedia se ubican en Estados Unidos de América, España e Inglaterra. Por el contrario, la mayor parte de las publicaciones que usan el término cross-media proviene de la República Popular China, Estados Unidos de América, Alemania, Japón, Inglaterra e Italia.

La cross-medialidad es un campo de conocimiento muy débil en el Cono Sur. Esto puede ser explicable a la luz de la formación de colegios invisibles por la relación académica frecuente entre América Latina y España, además del uso del idioma, ya que un 98 \% de la producción académica alrededor del término cross-media está escrita en inglés.

En cuanto a las áreas de conocimiento se encontró que nueve de las diez áreas destacadas en torno al término transmedia son del ámbito cultural y social, mientras que cinco de las diez áreas destacadas en torno al término cross-media presentan un interés común por los aspectos técnicos, donde se destaca el área de la computación. Sin embargo, en el campo de los estudios de la comunicación se encuentran 
muchas coincidencias en el uso de los términos transmedia y crossmedia. A menudo ambas nociones son tomadas como sinonimias y comparten autores de referencia como Henry Jenkins.

Los datos recogidos en esta investigación muestran la necesidad de abrir el espectro para entender la comunicación transmedia y cross-media como objetos de estudio afines que deben ser estudiados de forma interdisciplinar. Está claro que en lugar de estar buscando diferencias entre comunicación cross-media y transmedia, como ha sido habitual en los estudios sobre la comunicación, es importante entender lo fértil que resulta observar el concepto cross-media como una noción que tiene un origen multidisciplinar, y que no se agota en los medios y la narrativa como la hemos pretendido entender cuando la identificamos únicamente como un concepto adyacente a la comunicación transmedia. Es evidente que, después de una década de producción de conocimiento sobre el campo de la comunicación transmedia, se percibe un agotamiento en el tipo de investigaciones sobre estudios de caso, intentando identificar cómo la industria ha logrado expandir universos narrativos. Detectamos un terreno expedito para otro tipo de investigaciones que lleven lo transmedial a otros escenarios (Montoya y Vásquez, 2018) donde se superen los entornos mediáticos; investigaciones desde la frontera de los conceptos en la cual la noción cross-media ofrece nuevos acercamientos al problema.

En lo que respecta a la metodología empleada en esta investigación, la bibliometría resulta especialmente útil como instrumento para dar cuenta de un fenómeno en las múltiples tradiciones académicas en las que ha sido analizado. Sin embargo, para futuras investigaciones es preciso desarrollar un análisis cualitativo más detallado de los usos concretos de las nociones de transmedialidad y crossmedialidad en los artículos de la muestra, así como de las metodologías que se han empleado en dichas investigaciones.

Finalmente, los resultados de la investigación abren nuevas preguntas e invitan a considerar la comunicación en un terreno amplio en el que los medios presentan cada vez más relaciones y se requieren diversas herramientas teóricas y metodológicas para comprender su complejidad $\mathbf{I}$ 


\section{Referencias}

Aarseth, E. (2006). The culture and business of cross-media productions. Popular Communication, 4(3), 203-211. https://doi.org/10.1207/ s15405710pc0403_4

Bechmann Petersen, A. (2009). Crossmedia: Innovation Networks for Traditional Media Organizations. [Tesis doctoral, Universidad de Aarhus].

Bolter, J. D., \& Grusin, R. (2002). Remediation: Understanding New Media (5. ${ }^{\mathrm{a}}$ reimp.). MIT Press.

Cambridge Dictionary (2011). Cross.media. En Cambridge Business English Dictionary (p. 195). Cambridge University Press, https://bit.ly/2Dn7avE

Couldry, N., Livingstone, S., \& Markham, T. (2010). Media Consumption and Public Engagement: Beyond the Presumption of Attention. Palgrave MacMillan.

Cronin, J. (2016). Teach students to communicate a brand story with transmedia storytelling. Journal of Research in Interactive Marketing, 10(2), 86-101. https://doi.org/10.1108/JRIM-01-2015-0004

Dailey, L., Demo, L., \& Spillman, M. (2005). The convergence continuum: A model for studying collaboration between media newsrooms. Atlantic Journal of Communication, 13(3), 150-168. https://doi. org/10.1207/s15456889ajc1303_2

De Solla Price, D. J. (1965). Networks of scientific papers. Science, 149(3683), 510-515. https://bit.ly/2Xlnqo3

Dena, C. (2004). Current state of cross media storytelling: preliminary observations for future design. En Presented in the European Information Systems Technologies Event [The Hague, Netherlands]. https://bit. ly/3i3BquH

Elleström, L. (2017). Transfer of media characteristics among dissimilar media. Palabra Clave, 20(3), 663-685. http://dx.doi.org/10.5294/ pacla.2017.20.3.4

Erdal, I. J. (2008). Cross-media News Journalism. Institutional, Professional and Textual Strategies and Practices in Multi-platform News Production. [Tesis doctoral, Universidad de Oslo]. https://bit.ly/2xLHiE2

Erdal, I. J. (2009). Cross-media (Re)Production Cultures. Convergence, 15(2), 215-231. https://doi.org/10.1177/1354856508105231 
Evans, E. J. (2011). Transmedia Television: Audiences, New Media and Daily Life. Routledge.

Ford, S. (2007, abril 5). Transmedia journalism: A story-based approach to convergence. Convergence Culture Consortium. FoE. Futures of Entertainment. https://bit.ly/317jMR9

Garfield, E. (1985). The geography of science: Disciplinary and national mapping. Journal of Information Science, 11(4), 147-159. https:/doi. org/10.1177/016555158501100402

Genette, G. (1989). Palimpsestos: La literatura en segundo grado (C. F. Prieto, Trad.). Taurus.

Giovagnoli, M. (2011). Transmedia Storytelling: Imagery, Shapes and Techniques. ETC Press.

Gosciola, V. (2012). Narrativa transmídia: conceituação e origens. En C. Campalans, D. Renó, y V. Gosciola. (Eds.), Narrativas Transmedia: Entre teorías y prácticas (pp. 7-14). Editorial Universidad del Rosario.

Hasebrink, U, \& Hepp, A. (2017). How to research cross-media practices? Investigating media repertoires and media ensembles. Convergence, 23(4), 362-377. https://doi.org/10.1177/1354856517700384

Hasebrink, U., \& Domeyer, H. (2012). Media repertoires as patterns of behaviour and as meaningful practices: A multimethod approach to media use in converging media environments. Participations. Journal of Audience $\mathcal{E}$ Reception Studies, 9(2), 757-779. https://bit.ly/2PpbqN1

Hills, M. (2016). LEGO Dimensions meets Doctor Who: Transbranding and New Dimensions of Transmedia Storytelling? Revista Iconol4 Revista científica de Comunicación y Tecnologías Emergentes, 14(1), 8-29. https://bit.ly/2DvAyA4

Hjarvard, S. (2008). The Mediatization of society: A theory of the media as agents of social and cultural change. Nordicom Review, 29(2), 105-134. https://bit.ly/2z7UULF

Ibrus, I., \& Scolari, C. (2012). Crossmedia Innovations. Texts, Markets, Institutions. Peter Lang.

Jenkins, H. (2001, junio 1). Convergence? I diverge. MIT Technology Review. https://bit.ly/256Jj7r 
Jenkins, H. (2003a, enero 15). Transmedia Storytelling: Moving characters from books to films to video games can make them stronger and more compelling. MIT Technology Review. https://bit.ly/22vlyRK

Jenkins, H. (Ed.). (2003b). Rethinking Media Change: The Aesthetics of Transition. MIT Press.

Jenkins, H. (2006). Convergence Culture: Where Old and New Media Collide. New York University Press.

Jensen, K. B. (1999). Intertextualities and intermedialities. En I. Bondebjerg \& H. K. Haastrup (Eds.), Intertextuality and Visual Media (pp. 63-85). University of Copenhague.

Kjus, Y. (2008). Event Media. Television Production Crossing Media Boundaries. [Tesis doctoral, Universidad de Oslo]. https://bit.ly/2PicHp4

Krotz, F. (2008). Media connectivity: Concepts, conditions and consequences. En A. Hepp, F. Krotz, S. Moores, \& C. Winter (Eds.), Connectivity, Networks and Flows. Conceptualizing Contemporary Communications (pp. 13-33). Hampton Press.

Lievrouw, L.A. (1989). The invisible college reconsidered: Bibliometrics and the development of scientific communication theory. Communication Research, 16(5), 615-628. https://doi.org/10.1177/009365089016005004

Livingstone, S. (2009). On the mediation of everything: ICA presidential address 2008. Journal of Communication, 59(1), 1-18. https://doi. org/10.1111/j.1460-2466.2008.01401.x

Molina, J. J. (2009). Panorama de la investigación en redes sociales. Redes. Revista Hispana para el Análisis de Redes Sociales, 17(11), 235-256. https://bit.ly/2Vil7OG

Montoya Bermúdez, D. F. y Vásquez Arias, M. (2018). Modelos para el diseño de experiencias transmedia en entornos educativos. Exploraciones en torno a The Walking Dead, La Odisea y Tom Sawyer. Revista Mediterránea de Comunicación, 9(1), 197-216. https://doi.org/10.14198/ MEDCOM2018.9.1.12

Negroponte, N. (2000 [1979]). El mundo digital: Un futuro que ya ha llegado. Ediciones B. 
Okazaki, A., Hairong, L., \& Hirose, M. (2012). Benchmarking the Use of QR Code in Mobile Promotion Three Studies in Japan. Journal of Advertising Research, 52(1) 102-117. https://dx.doi.org/10.2501/JAR-52-1-102-117

Pavlik, J. V. (2005). El Periodismo y los nuevos medios de comunicación. Paidós.

Peñafiel Sáiz, C. (2016). Reinvención del periodismo en el ecosistema digital y narrativas transmedia. AdComunica, (12), 163-182. http:// dx.doi.org/10.6035/2174-0992.2016.12.10

Renó, D. P. (2011). Periodismo, redes sociales y transmediación. Razón y Palabra, 16(78), 1-11. https://bit.ly/3hd36x2

Repiso, R., Jiménez-Contreras, E., y Aguaded, I. (2017). Revistas Iberoamericanas de Educación en SciELO Citation Index y Emerging Source Citation Index. Revista Española de Documentación Científica, 40(4), 1-13. http://dx.doi.org/10.3989/redc.2017.4.1445

Sanz Menéndez, L. (2003). Análisis de redes sociales: o como representar las estructuras sociales subyacentes. Apuntes de Ciencia y Tecnología, (7), 20-29. https://bit.ly/2ULmlH1

Scolari, C. A. (2009). Transmedia storytelling: Implicit consumers, narrative worlds, and branding in contemporary media production. International Journal of Communication, (3), 586-606. https://bit.ly/2SaZ8Zd

Scolari, C. A. (2013). Narrativas transmedia: Cuando todos los medios cuentan. Deusto.

Scolari, C. A., Jiménez, M., y Guerrero, M. (2012). Narrativas transmediáticas en España: cuatro ficciones en busca de un destino cross-media. Comunicación y Sociedad, 25(1), 137-163. http://hdl. handle.net/10171/27363

Scolari, C., Lugo Rodríguez, N., y Masanet, M. J. (2019). Educación transmedia. De los contenidos generados por los usuarios a los contenidos generados por los estudiantes. Revista Latina de Comunicación Social, (74), 116-132. https://bit.ly/3g5zmks

Simons, N. (2014). Audience reception of cross-and transmedia TV drama in the age of convergence. International Journal of Communication, (8), 2220-2239. https://bit.ly/2vgt0JP 
Singer, J. B. (2004). Strange bedfellows? The diffusion of convergence in four news organizations. Journalism Studies, 5(1), 3-18. https://doi. org/10.1080/1461670032000174701

Solano-López, E., Castellanos Quintero, S., López Rodríguez del Rey, M., y Hernández Fernández, J. (2009). La bibliometría: una herramienta eficaz para evaluar la actividad científica postgraduada. Medisur, 7(4), 59-62. https://bit.ly/3kXu11Z

Thomson Reuters (2008). Using Bibliometrics. A guide to evaluating research performance with citation data [White Paper]. http://ips. clarivate.com $/ \mathrm{m} / \mathrm{pdfs} / 325133$ _thomson.pdf

Veglis, A. (2012). From Cross Media to Transmedia Reporting in Newspaper Articles. Publishing Research Quarterly, 28(4), 313-324. https://doi. org/10.1007/s12109-012-9294-z

Villa, M. I. (2011). Producción Cross-media. El caso de Televisió de Catalunya. [Tesis doctoral, Universidad Autónoma de Barcelona]. https://bit.ly/2GwfdoR

Weedon, A., Miller, D., Franco, C. P., Moorhead, D., \& Pearce, S. (2014). Crossing media boundaries: Adaptations and new media forms of the book. Convergence, 20(1), 108-124. https://doi. org/10.1177/1354856513515968

Wohlwend, K. E. (2012). The boys who would be princesses: Playing with gender identity intertexts in Disney Princess transmedia. Gender and Education, 24(6), 593-610. https://doi.org/10.1080/09540253.2012.674495

Wu, F., Jiang, X, Li, X., Tang, S., Lu, W., Zhang, Z., y Zhuang, Y. (2015). Cross-Modal Learning to Rank via Latent Joint Representation. IEEE Transactions on Image Processing, 24(5), 1497-1509. https://doi. org/10.1109/TIP.2015.2403240 